\title{
Therapeutic Hypothermia after Pediatric Cardiac Arrest Trials: The Vanguard Phase Experience and Implications for Other Trials
}

Victoria L. Pemberton, RNC, MS ${ }^{1}$, Brittan Browning, MS, RD², Angie Webster, $\mathbf{M S t a t}^{2}$, J. Michael Dean, MD, MBA ${ }^{2}$, and Frank W. Moler, MD, MS $^{3}$

${ }^{1}$ National Heart, Lung, and Blood Institute, NIH

${ }^{2}$ University of Utah

${ }^{3}$ University of Michigan

\section{Abstract}

Objective-To determine whether an 18-month vanguard phase, in the Therapeutic Hypothermia after Pediatric Cardiac Arrest (THAPCA) trials, confirmed study feasibility and patient safety, a prerequisite to continued funding by the sponsor.

Design-Randomized controlled trial

Setting-Pediatric intensive care and pediatric cardiac care units in 15 clinical sites in the United States and Canada

Patients-Children, aged 48 hours to 18 years of age, with return of circulation after cardiac arrest

Interventions-Therapeutic hypothermia versus therapeutic normothermia

Measurements and Main Results-The first 15 of 20 potential sites to obtain IRB and subcontract approvals were selected as vanguard sites. IRB approvals were obtained 92 days (median, interquartile range [IQR] 65-114) and subcontracts signed 34 days (IQR 20-48) after distribution. Sites screened subjects 13 days (IQR 9-21) and enrolled the first subjects 64 days (IQR 13-154) after study launch. The recruitment milestone was reached four months ahead of schedule with no safety concerns identified. Overall recruitment in this ongoing trial remains on target.

Conclusions-The THAPCA vanguard phase proved beneficial for the investigators and funding agency. Since complex multicenter trials are rarely ready to launch when grant funds are received, the vanguard allowed time to refine the protocol and recruitment approaches. Competition for vanguard positions led to expedient IRB and subcontract completion. Early

Victoria Pemberton, RNC, MS, NHLBI, NIH, Division of Cardiovascular Sciences, 6701 Rockledge Drive, Room 8102, Bethesda, Maryland, 20892, Phone: 301-435-0510, Fax: 301-480-2858, pembertonv@nhlbi.nih.gov.

Institutions where the trials were conducted during the Vanguard Phase:

Arkansas Children's Hospital, Children's Hospital of Michigan (Detroit), Children's Hospital of New York, Children's Hospital of Philadelphia, Children's National Medical Center, Cincinnati Children's Hospital, Hospital for Sick Children in Toronto, University of Utah and Primary Children's Medical Center, Children's Medical Center Dallas, Children's Memorial at Baylor, Children's Memorial Hospital at Northwestern, Seattle Children's Hospital, University of California LA and Children's Hospital of Los Angeles, University of Pittsburgh Medical Center, Washington University and St. Louis Children's Hospital, University of Michigan (Ann Arbor)

No reprints will be ordered

Disclosures: None 
success and sustained momentum contributed to recruitment at or above goals. Financial risks to the sponsor were minimized by tying funding for the full trial to achieving pre-specified milestones. A vanguard phase may be a desirable strategy for the successful conduct of other complex clinical trials.

Clinical Trial Registration-NCT00880087 and NCT00878644 http://clinicaltrials.gov/ct2/ show/NCT00880087?term=pediatric+hypothermia\&rank=4 http://clinicaltrials.gov/ct2/show/ NCT00878644?term=pediatric + hypothermia\&rank $=5$

\section{MeSH terms}

pediatric intensive care; cardiopulmonary resuscitation; induced hypothermia

\section{Introduction}

Conducting multicenter clinical research is challenging, particularly when the intervention has a short therapeutic window and is implemented in critically ill children who have experienced an unanticipated, life-threatening event. Administrative challenges (multiple Institutional Review Board [IRB] approvals, subcontracts, and training), scientific challenges (equipoise and standardizing protocols among various centers) and financial challenges are common in multi-site trials $(1,2)$. These are compounded when pediatric researchers must identify eligible research subjects from smaller pediatric patient pools compared with adult trials, address complex issues of parental consent and child assent (3, 4), tackle the perceptions and lack of understanding among parents and pediatric healthcare providers about research with children (5-7), manage the range of IRB assessments of trial risks and benefits $(4,8,9)$, and procure the requisite expertise and additional funds needed to perform study interventions and long-term follow-up in children (10). Strategies have been identified for simplifying research processes, streamlining activities and decreasing the often-excessive time required to complete a trial (11-14), yet investigators continue to struggle to complete recruitment on time and within the budget allotted $(15,16)$. Given the hurdles for complex pediatric trials, implementing strategies and practices that can enhance trial success is paramount.

One potential strategy is a vanguard phase, sometimes referred to as a pilot or run-in phase. Vanguards have been used to assess the safety of an intervention, to determine the feasibility of a trial, or establish the ability of a study to meet treatment or recruitment goals. Although logistics and inclusion/exclusion criteria are usually considered finalized when protocols are completed, vanguards offer the opportunity to re-examine these in a real world setting and make proper adjustments prior to launching the main trial, thereby increasing the likelihood of trial success (17).

In 2000, the Action to Control Cardiovascular Risk in Diabetes (ACCORD) trial, funded by the National Heart, Lung, and Blood Institute (NHLBI), used a vanguard phase to determine recruitment and treatment feasibility. Recruitment of 1174 subjects in 20 weeks exceeded the target goal of 1000, demonstrating feasibility. The successful vanguard recruitment strategies identified permitted the main trial to complete target enrollment of 10,000 subjects within 3 months of the planned date (18). The vanguard phase also facilitated site preparedness and identified protocol modifications that enhanced study feasibility (19).

In 2007, investigators for the Therapeutic Hypothermia after Pediatric Cardiac Arrest (THAPCA) trials approached the NHLBI to request permission to submit an application for a 30-site randomized clinical trial. Based on prior cooling studies that demonstrated improved functional survival in adults after cardiac arrest and in preterm infants with birth asphyxia (20-25), THAPCA would test the hypothesis that therapeutic hypothermia versus 
therapeutic normothermia would improve survival and neurobehavioral outcomes after cardiac arrest in two separate clinical trials: in-hospital (IH) and out-of-hospital (OH).

\section{Why a Vanguard Phase?}

NIH peer review reflected enthusiasm for THAPCA and the potential for understanding optimal pediatric temperature management that might lead to improved outcomes, given the limited therapeutic options available. The NHLBI Advisory Council, the second level of review for all proposed grants, also recognized the importance of answering this question. However, there were concerns about the proposed costs and complexities of the trials, the absence of pilot outcome data using the specific THAPCA protocol, the feasibility of obtaining parental consent and initiating the therapy within the narrow therapeutic window in a critically ill pediatric population, the ability to obtain primary outcome data on patients with significant disability or in long-term care at 12 months, the agreement and cooperation of the various specialists to enroll patients and whether there was equipoise regarding the study interventions.

In response, the NHLBI agreed to fund an 18-month vanguard phase during which time the study was required to meet pre-specified milestones and demonstrate participant safety and study feasibility in order for NHLBI to consider financial support for the full trial. The goals of the THAPCA vanguard phase were to:

1. Provide six months to complete administrative activities for study launch.

2. Limit financial exposure by providing funds for 18 months, recruiting at half the number of proposed sites and basing future funding on achieving pre-specified milestones.

3. Establish the feasibility of recruiting, consenting parents and initiating the study intervention within six hours in critically ill children being cared for by multiple subspecialists.

4. Demonstrate the ability to obtain primary outcome data.

5. Ensure that the trials could be conducted safely.

6. Identify operational efficiencies with the potential to enhance study success.

Near the end of the enrollment period, an administrative review of the study was planned to evaluate feasibility, progress and safety, assess continued relevance of the scientific questions and determine the trial's future.

This paper presents the lessons learned and challenges of implementing a vanguard phase in a complex trial in critically ill children and provides information to help investigators determine whether a vanguard phase might be an appropriate approach for other complex clinical trials.

\section{Materials and Methods}

\section{Original Design}

The investigators originally proposed two prospective randomized controlled trials to test the effectiveness of therapeutic hypothermia versus therapeutic normothermia after inhospital (THAPCA-IH) or out-of-hospital (THAPCA-OH) cardiac arrest in children. Separate trials were proposed because arrest etiologies and patient outcomes of the two groups are substantially different (26). The primary endpoint, survival with good neurobehavioral outcome at 12 months, is assessed using the Vineland Adaptive Behavior 
Scales. Subjects (834 for both trials) were expected to be enrolled over four and a half years at 30 clinical sites.

\section{Vanguard Phase Design}

The investigators and NHLBI staff developed a timeline and budget, and set milestones for the vanguard phase which began in March of 2009. The first six months were set aside for activities preparatory to launching the study followed by 12 months for recruitment at 15 sites (Figure 1). The enrollment milestone was set at 50 patients, all of whom would be analyzed as part of the total study population. The trials were registered in ClinicalTrials.gov and IRB approval was obtained at all enrolling centers.

\section{Vanguard Site Selection}

In addition to the THAPCA PI site, 20 sites were invited to participate. Most of these sites came from two experienced pediatric clinical trial research Networks: the Health Resources and Services Administration (HRSA)-funded Pediatric Emergency Care Applied Research Network (PECARN) (27) and the Eunice Kennedy Shriver National Institute of Child Health and Human Development (NICHD)-funded Collaborative Pediatric Critical Care Research Network (CPCCRN) (28). The PI site and one other site obtained IRB approval first and shared their regulatory documents with the remainder of the sites to facilitate subsequent IRB approvals. Standardized contracts were sent to all sites. Site PIs and contract offices were given a firm deadline by which to provide institutional approvals. The first 15 sites to obtain IRB approval and finalize subcontracts became vanguard sites.

\section{Results}

During the six-month planning phase, the protocol and supporting documentation was completed, and the Data and Safety Monitoring Board met to review and approve the study protocol. Table 1 shows key study activities occurring during the vanguard phase. IRB approvals were obtained 92 days (median, interquartile range [IQR] 65-114) and subcontracts signed 34 days (IQR 20-48) after distribution. At month five, 14 main sites and 2 alternate sites received study training. (One site withdrew from study participation prior to training.) At month six, the 12-month recruitment phase began at 15 sites with the first patients screened a median of 13 days (IQR 9-21) later. From study launch, the median time to enroll the first patient across all sites was 64 days (IQR 13-154 days) with one site never enrolling. This site was discontinued from study participation at 226 days.

Recruitment remained on target during the initial six months (Figure 2) and accelerated quickly thereafter. Subjects (92\%) were consented and randomized within the 6-hour timeframe as mandated by the protocol. The median time to therapy initiation was 5.6 hours (IQR 4.6-6.2). Study monitoring revealed that similar numbers of patients were eligible for the $\mathrm{IH}(86)$ and $\mathrm{OH}$ trials (88), yet fewer were approached for the IH study (76\% versus $91 \%$ ) and fewer consented (49\% versus $66 \%$ ). The DSMB met regularly during the vanguard, reviewed blinded data and had no safety concerns. The minimum vanguard phase recruitment threshold of 50 subjects was crossed four months ahead of schedule.

Table 2 shows the four protocol amendments that were implemented during the vanguard period to enhance study eligibility/enrollment and decrease study burden. These changes, based on experience at the sites, were approved by the study Executive Committee, the DSMB, and each site's IRB. The median number of days for IRB approval of each of the four protocol amendments was 36, 34, 29 and 43 days.

The rate of successful primary outcome measurement at 12 months with the Vineland Adaptive Behavioral Scales (VABS) could not be obtained due to the short duration of the 
vanguard phase. Since the VABS were also being used to measure three-month outcomes, this was used as a surrogate measure of the ability to obtain the primary outcome, with a follow-up rate of $100 \%$.

\section{Discussion}

The THAPCA vanguard phase proved to be a good strategy and demonstrated that the study was feasible, safe and could be implemented efficiently and effectively. Efficiency was enhanced by allocating six months for pre-trial launch activities, and implementing deadlines for contract and IRB approvals at 20 sites that competed for 15 vanguard positions. Effectiveness was enhanced by the opportunity to 1) test the inclusion and exclusion criteria and to make agile changes to the protocol before expanding the number of sites; 2 ) to examine the real-world implementation of the study procedures and clarify areas of misunderstanding; 3 ) to assess the willingness of parents and healthcare providers of the study population to provide consent; and 4) to learn optimal ways to adjust study resources for high or low enrolling sites. Many of the successful strategies and lessons learned during the vanguard have been adopted during the main trial, enhancing success. Ultimately the success of a vanguard is measured by whether the main trial finishes on time and with sufficient participants; at the time of this paper, THAPCA is recruiting on target (Figure 3).

\section{Factors for Success}

Launching clinical trials requires orchestrating many activities and rarely is a study ready to enroll the first patient upon receipt of funding although this is how most federal funding of trials is structured. The THAPCA investigators were well-prepared when they approached NHLBI about funding. The PIs presented pilot data based on a similar protocol, nearly final study materials and an established trial infrastructure that had been developed from previous grant funding. Pilot data collection (R21 HD044955) demonstrated that a hypothermia trial was feasible in both out-of-hospital and in-hospital arrest settings $(29,30)$. A NICHD planning grant (R34 HD050531) funded the development of documents (protocol, consent forms) necessary for the execution of a multicenter clinical trial. The THAPCA data coordinating center (DCC) supported the research activities in both the PECARN and CPCCRN networks from their inception and used similar study tools, forms and procedures, thereby enhancing efficiency. Despite these extensive preparations, the vanguard phase permitted time to finalize the study documents, obtain IRB and subcontract approvals, and identify and train sites.

THAPCA clinical sites obtained IRB approval efficiently. The 20 clinical centers from the two Networks were highly motivated to obtain IRB approval, which occurred as early as 56 days and no longer than 140 days after submission. This compares favorably to other NIH funded trials. For example, the time required to obtain central IRB approval in Phase III clinical trials in the National Cancer Institute's Cancer Therapy Evaluation Program ranged from 46-230 days (31). Finalizing subcontracts, which often requires more time than IRB approval, occurred quite expeditiously in one to 53 days (Table 1).

Some unanticipated benefits accrued from having a smaller number of sites in the vanguard phase than would have been the case when launching the full trial. The sites expressed satisfaction in receiving more individualized attention during the extensive three-day training session and after trial launch. In addition, the burden on the principal THAPCA investigators to guide each site through its first two randomizations was limited by having fewer sites initially. Once the trial was underway, protocol deviations could be observed more easily and errors corrected more quickly. 


\section{Study Conduct}

The vanguard recruitment period confirmed that the clinical sites could consent and randomize subjects within the required six-hour window. This was considered to be a challenge particularly for children whose parents may be delayed in getting to the hospital or in such emotional distress as to render informed consent difficult. Informed consent requires parents to distill complex information about their child's medical condition, the prognosis and treatment plan as well as the details of the proposed research. In THAPCA, this was complicated by the unexpectedness of the cardiac arrest event and parents being approached by a member of the research team, an individual the family had likely never met, in the turbulent environment of an emergency room or critical care unit.(32-34)

A primary objective of the vanguard was to demonstrate that the THAPCA protocol could be implemented safely. The investigators adopted a strategy for reporting adverse events based on the "sentinel event" approach taken by the Pediatric Heart Network's Single Ventricle Reconstruction Trial investigators (35). The system relies on closely monitoring a small number of clinically relevant, serious events thereby limiting the volume of data (or noise) to be managed. The DSMB met regularly during the vanguard phase, reviewed blinded study and safety data and found that subjects with the most serious events were being properly identified and monitored. The vanguard period confirmed that this reporting approach allowed the medical monitor, the DSMB Chair and NHLBI staff to focus attention on the most important events without sacrificing subject safety.

Inclusion and exclusion criteria are not always fully tested when trials are proposed and the vanguard phase allowed these to be refined. For example, ten patients with a repeat cardiac arrest prior to randomization were excluded from the study early in the vanguard phase, but review of their mortality rate indicated that this population was not intrinsically moribund (the original purpose of the exclusion criterion) and therefore they could be included, increasing the potential recruitment pool. Four protocol amendments were approved during the vanguard year compared to only one in the year following full study launch. Amendment approvals across fewer sites decreased administrative burden on the study, were obtained expeditiously (Table 2) and were already incorporated into the protocol when the full complement of study sites was brought on.

It would have been ideal to determine the feasibility of having the surviving subjects return for measurement of the primary outcome, 12-month survival with good neurobehavioral status. But this was not possible due to the short duration of the vanguard phase. However, the three-month outcome data in $100 \%$ of the surviving study subjects was obtained, which was considered an encouraging surrogate measure of the ability to obtain the 12-month endpoint. This has been borne out in the full trial: at this time, the follow-up rate for the 12 month primary outcome measure is $95 \%$ of eligible subjects.

\section{Momentum and Equipoise}

Momentum is a significant contributor to a successful trial; however, recruitment usually ramps up slowly after studies launch, particularly in trials involving rare events. Due to the planning period and competition, the THAPCA vanguard sites were well-prepared and motivated, building early momentum for the trial which resulted in the study achieving target enrollment even in the first few months after launch. Eleven of the 15 sites enrolled a patient within three months of study launch (Figure 2).

Maintaining momentum is equally important in a trial and the compressed timeline of the vanguard phase required creative strategies be used and implemented quickly. Monthly PI calls with discussions about recruitment and lessons learned, site recognition for each randomization, a webinar on effective consenting techniques for the population under study 
and individual calls to sites were conducted. Study teams were also aware of "back-up sites" that were eager and ready to begin the trial should a center fail to perform adequately. By meeting enrollment targets four months ahead of schedule, early administrative review occurred with approval to continue the study, avoiding interruptions in study activities.

Children in THAPCA are clinically managed by a number of different specialists. The vanguard phase allowed study leadership to develop collaborations with clinical colleagues and assess the willingness of patient care providers to randomize children into the trial. During the 2003 pilot study, only 3\% of children with cardiac arrest were being treated with hypothermia. Data collected on off-study clinical hypothermia use during the vanguard phase showed that $28 \%$ of eligible patients were receiving this therapy in the IH cohort and $24 \%$ in the $\mathrm{OH}$ cohort. Recognizing this early allowed the study leadership to increase surveillance of off-study hypothermia and provide counseling to study PIs on managing this challenge to equipoise at their sites.

The vanguard phase began to reveal a pattern of lower IH enrollment compared to $\mathrm{OH}$ recruitment. Similar numbers of patients were study-eligible but fewer were approached and consented for the IH study. For example, although pilot data didn't demonstrate that enrolling infants and children undergoing cardiac surgery would be particularly challenging, the vanguard showed that some centers used hypothermia (deep hypothermic circulatory arrest) during surgery and expressed concern about the risk of bleeding with hypothermia. In addition, it was learned that sites that did employ hypothermia had a variety of strategies and target temperatures for cooling $(36,37)$. While no definitive studies have been conducted of hypothermia in children after cardiac surgery, the vanguard allowed these issues to be identified so that strategies for addressing equipoise and recruiting in the cardiac intensive care units could be explored.

\section{Financial Implications}

The funds NHLBI provided for the vanguard phase represented a small fraction of the total cost of the full trial. The success of the vanguard phase was evaluated in an administrative review conducted by NHLBI leadership, which focused on recruitment, participant safety, study feasibility and the continued relevance of the study question. Based on the success of the THAPCA vanguard in all areas, the decision was made to fund the full trial with a greater measure of certainty about the outcome. This represents a "real-options" approach to risky investment where a project can be terminated after a technical failure (38). For example, a pharmaceutical company may use this approach to estimate the potential value and investment needed to bring a new product to market. There is likely to be uncertainty in the early stages (before the product has undergone extensive testing) at which time the company must decide whether to continue investment or end the project. The real options model calculates the value based on multiple options at critical time points (39). Clinical trials are risky by their very nature, and the financial value of the option to discontinue a trial may allow more effective allocation of research funding, particularly during times of fiscal uncertainty.

The study leadership used the vanguard phase to assess budgets and reallocate funds as needed for the success of the full trial. Low enrolling centers forfeited infrastructure funds, which were then used to bring on additional sites during the main trial and to purchase extra cooling blankets needed for high enrolling centers.

\section{Vanguard challenges}

Implementing a vanguard was not without challenges. The relatively tight timeline and anticipation of the administrative review required a more intensive schedule of monitoring 
and investigator calls than otherwise would have been the case. The investigators also faced financial challenges. The overall timeline of the study was extended by 12 months yet only those funds originally requested in the grant proposal were provided. Many institutions require a viable long-term funding plan before allowing new positions to be created, so THAPCA vanguard sites were challenged to allocate experienced staff to the project without the assurance of funding beyond the vanguard phase. Finally, the vanguard phase extended the planned overall study timeline. This poses a potential risk that the intervention could be more widely adopted for clinical use outside of a trial, rendering trial recruitment more difficult. Extended study duration also increases the potential for trial fatigue among the enrolling sites. For NHLBI, the challenges included adapting the standard 5-year funding approach to add the additional time for the vanguard and developing criteria for a substantive administrative review of vanguard progress.

\section{Conclusions}

The THAPCA vanguard phase has proven beneficial for both the funding agency and the THAPCA investigators by providing extra time that was essential for launching this trial and for implementing changes that contributed to successful enrollment. In reality, complex multicenter trials such as this one are not ready to launch at the moment that grant funds are received and additional time is often needed to fine-tune the protocol and recruitment approaches. Lessons learned during the THAPCA vanguard phase were shared with new sites as they were brought on for the remainder of the trial, thereby allowing them to avoid pitfalls and adopt best practices. Overall the pros outweighed the cons of implementing a vanguard phase in the THAPCA trial and we believe a similar approach may be of benefit for successful completion of other large clinical trials.

\section{Acknowledgments}

Funding Sources: NIH U01 HL094339 and U01 HL094345

We wish to thank Gail D. Pearson, MD, ScD for her expert guidance during vanguard study design and manuscript preparation.

Participating vanguard sites:

University of Michigan, Ann Arbor, MI (F. Moler, M.D., M.S.)

Children's Hospital of Philadelphia, Philadelphia, PA (A. Topjian, M.D)

Children's National Medical Center, Washington D.C. (J. Berger M.D.)

Children's Hospital of Michigan, Detroit, MI (K. Meert, M.D.)

University of Pittsburgh, Pittsburgh, PA (J. Carcillo/E. Fink, M.D.)

Children's Hospital of LA (USC/UCLA), LA, CA (C. Newth/R. Harrison, M.D.)

Washington University St. Louis Children's Hospital, (J. Pinada, M.D.)

Children's Hospital Medical Center, Cincinnati, Ohio (D. Wheeler, M.D.)

University of Utah - Primary Children's Medical Center (K. Statler, M.D.)

Children's Hospital of New York, Columbia University (C. Schleien, M.D.)

Children's Memorial Hospital, Chicago, IL (D. Goodman, M.D.)

University of Arkansas, Little Rock, AR (R. Sanders, M.D) 
Hospital for Sick Kids, Toronto, ON (J. Hutchinson, M.D.)

Children's Hospital Dallas, TX (J. Koch, M.D.)

Texas Children's Hospital (Baylor), Houston, TX (L. Loftis, M.D.)

\section{References}

1. Duley L, Antman K, Arena J, et al. Specific barriers to the conduct of randomized trials. Clin Trials. 2008; 5:40-48. [PubMed: 18283079]

2. Aban IB, Wolfe GI, Cutter GR, et al. The MGTX experience: challenges in planning and executing an international, multicenter clinical trial. J Neuroimmunol. 2008; 201-202:80-84.

3. Caldwell PH, Murphy SB, Butow PN, et al. Clinical trials in children. Lancet. 2004; 364:803-811. [PubMed: 15337409]

4. Field, M. Ethical Conduct of Clinical Research Involving Children. Washington, DC: National Academies Press; 2004.

5. Caldwell PH, Butow PN, Craig JC. Pediatricians' attitudes toward randomized controlled trials involving children. J Pediatr. 2002; 141:798-803. [PubMed: 12461496]

6. Singhal N, Oberle K, Burgess E, et al. Parents' perceptions of research with newborns. J Perinatol. 2002; 22:57-63. [PubMed: 11840244]

7. Singhal N, Oberle K, Darwish A, et al. Attitudes of health-care providers towards research with newborn babies. J Perinatol. 2004; 24:775-782. [PubMed: 15295610]

8. Sugarman J. Determining the appropriateness of including children in clinical research: how thick is the ice? JAMA. 2004; 291:494-496. [PubMed: 14747509]

9. Shah S, Whittle A, Wilfond B, et al. How do institutional review boards apply the federal risk and benefit standards for pediatric research? JAMA. 2004; 291:476-482. [PubMed: 14747505]

10. Li JS, Eisenstein EL, Grabowski HG, et al. Economic return of clinical trials performed under the pediatric exclusivity program. JAMA. 2007; 297:480-488. [PubMed: 17284698]

11. Califf RM. Clinical trials bureaucracy: unintended consequences of well-intentioned policy. Clin Trials. 2006; 3:496-502. [PubMed: 17170032]

12. Burman WJ, Reves RR, Cohn DL, et al. Breaking the camel's back: multicenter clinical trials and local institutional review boards. Ann Intern Med. 2001; 134:152-157. [PubMed: 11177319]

13. Dilts DM, Sandler AB. Invisible barriers to clinical trials: the impact of structural, infrastructural, and procedural barriers to opening oncology clinical trials. J Clin Oncol. 2006; 24:4545-4552. [PubMed: 17008693]

14. Dilts DM, Sandler AB, Cheng SK, et al. Steps and time to process clinical trials at the Cancer Therapy Evaluation Program. J Clin Oncol. 2009; 27:1761-1766. [PubMed: 19255315]

15. McDonald AM, Knight RC, Campbell MK, et al. What influences recruitment to randomised controlled trials? A review of trials funded by two UK funding agencies. Trials. 2006; 7:9. [PubMed: 16603070]

16. Schroen AT, Petroni GR, Wang H, et al. Preliminary evaluation of factors associated with premature trial closure and feasibility of accrual benchmarks in phase III oncology trials. Clin Trials. 2010; 7:312-321. [PubMed: 20595245]

17. Thabane L, Ma J, Chu R, et al. A tutorial on pilot studies: the what, why and how. BMC Med Res Methodol. 2010; 10:1. [PubMed: 20053272]

18. Buse JB, Bigger JT, Byington RP, et al. Action to Control Cardiovascular Risk in Diabetes (ACCORD) trial: design and methods. Am J Cardiol. 2007; 99:21i-33i.

19. Kingry C, Bastien A, Booth G, et al. Recruitment strategies in the Action to Control Cardiovascular Risk in Diabetes (ACCORD) trial. Am J Cardiol. 2007; 99:68i-79i. [PubMed: 17196465]

20. Gluckman PD, Wyatt JS, Azzopardi D, et al. Selective head cooling with mild systemic hypothermia after neonatal encephalopathy: multicentre randomised trial. Lancet. 2005; 365:663670. [PubMed: 15721471] 
21. Shankaran S, Laptook AR, Ehrenkranz RA, et al. Whole-body hypothermia for neonates with hypoxic-ischemic encephalopathy. N Engl J Med. 2005; 353:1574-1584. [PubMed: 16221780]

22. Mild therapeutic hypothermia to improve the neurologic outcome after cardiac arrest. N Engl J Med. 2002; 346:549-556. [PubMed: 11856793]

23. Bernard SA, Gray TW, Buist MD, et al. Treatment of comatose survivors of out-of-hospital cardiac arrest with induced hypothermia. N Engl J Med. 2002; 346:557-563. [PubMed: 11856794]

24. Eicher DJ, Wagner CL, Katikaneni LP, et al. Moderate hypothermia in neonatal encephalopathy: safety outcomes. Pediatr Neurol. 2005; 32:18-24. [PubMed: 15607599]

25. Eicher DJ, Wagner CL, Katikaneni LP, et al. Moderate hypothermia in neonatal encephalopathy: efficacy outcomes. Pediatr Neurol. 2005; 32:11-17. [PubMed: 15607598]

26. Moler FW, Meert K, Donaldson AE, et al. In-hospital versus out-of-hospital pediatric cardiac arrest: a multicenter cohort study. Crit Care Med. 2009; 37:2259-2267. [PubMed: 19455024]

27. [Accessed June 30, 2011] Pediatric Emergency Care Applied Research Network. 2011. Available online at: http://www.pecarn.org/

28. NICHD. [Accessed June 30, 2011.] Collaborative Pediatric Critical Care Research Network. 2011. Available online at: http://cpccrn.org/index.html

29. Meert KL, Donaldson A, Nadkarni V, et al. Multicenter cohort study of in-hospital pediatric cardiac arrest. Pediatr Crit Care Med. 2009; 10(5):544-553. [PubMed: 19451846]

30. Moler FW, Donaldson AE, Meert K, et al. Multicenter cohort study of out-of-hospital pediatric cardiac arrest. Crit Care Med. 2011; 39:141-149. [PubMed: 20935561]

31. Dilts DM, Sandler AB, Baker M, et al. Processes to activate phase III clinical trials in a Cooperative Oncology Group: the Case of Cancer and Leukemia Group B. J Clin Oncol. 2006; 24:4553-4557. [PubMed: 17008694]

32. Ungar D, Joffe S, Kodish E. Children are not small adults: documentation of assent for research involving children. J Pediatr. 2006; 149:S31-33. [PubMed: 16829240]

33. Natale JE, Joseph JG, Pretzlaff RK, et al. Clinical trials in pediatric traumatic brain injury: unique challenges and potential responses. Dev Neurosci. 2006; 28:276-290. [PubMed: 16943651]

34. Macrae D. Conducting clinical trials in pediatrics. Crit Care Med. 2009; 37(1 Suppl):S136-139. [PubMed: 19104213]

35. Virzi L, Pemberton V, Ohye RG, et al. Reporting adverse events in a surgical trial for complex congenital heart disease: the Pediatric Heart Network experience. J Thorac Cardiovasc Surg. 2011; 142:531-537. [PubMed: 21397260]

36. Brown DW, Connor JA, Pigula FA, et al. Variation in preoperative and intraoperative care for first-stage palliation of single-ventricle heart disease: a report from the Joint Council on Congenital Heart Disease National Quality Improvement Collaborative. Congenit Heart Dis. 2011; 6:108-115. [PubMed: 21426524]

37. Jonas RA. Deep hypothermic circulatory arrest: current status and indications. Semin Thorac Cardiovasc Surg Pediatr Card Surg Annu. 2002; 5:76-88. [PubMed: 11994867]

38. Willigers BJA, Hansen TL. Project valuation in the pharmaceutical industry: A comparison of least-squares Monte Carlo real option valuation and conventional approaches. $\mathrm{R}$ and $\mathrm{D}$ Management. 2008; 38:520-537.

39. Copeland T, Tufano P. A Real-World Way to Manage Real Options. Harvard Business Review. 2004; 82:90-99. [PubMed: 15029793] 


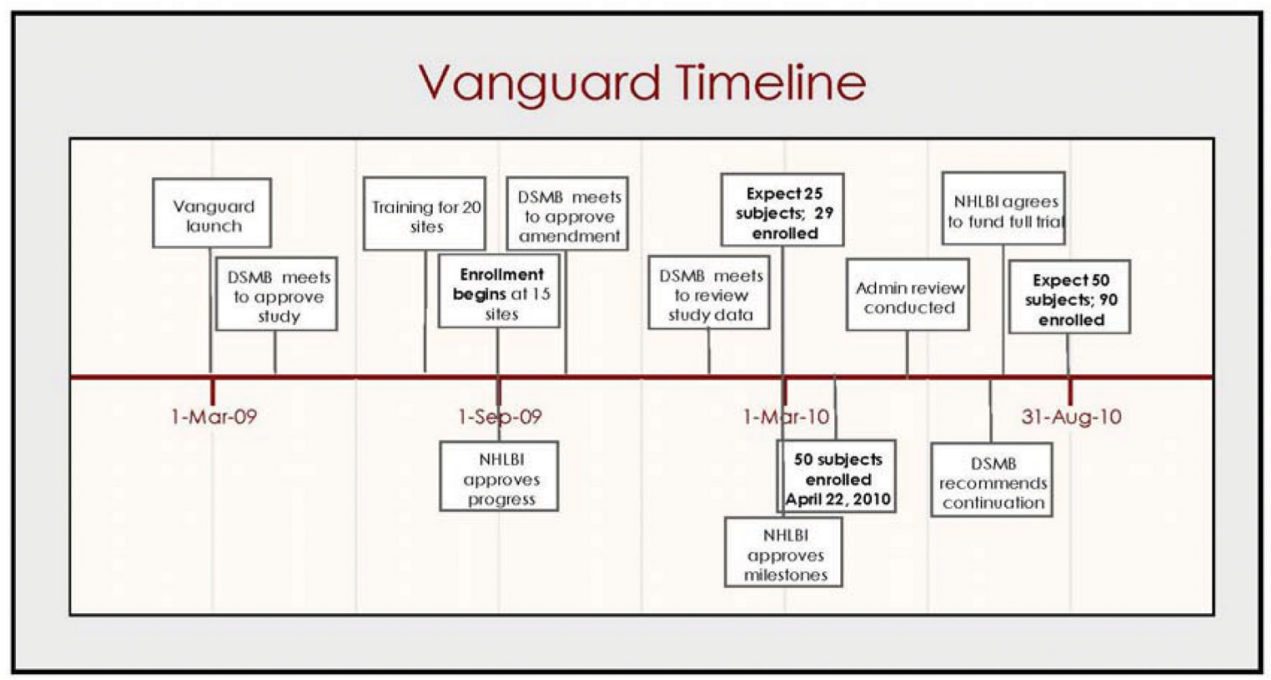

Figure 1.

Vanguard Timeline 


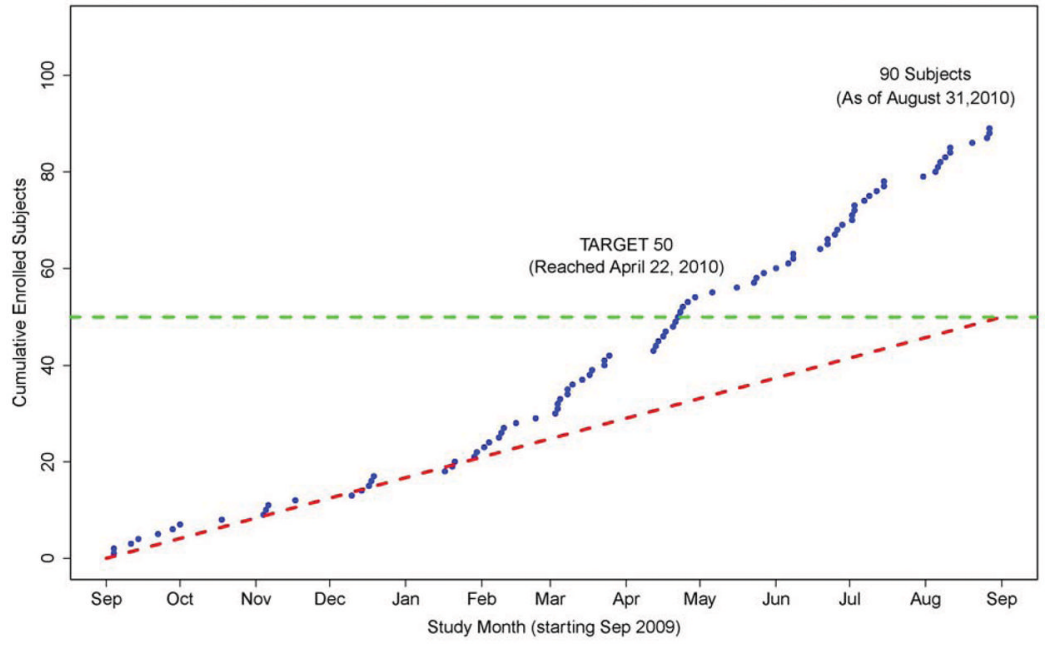

Figure 2.

THAPCA Vanguard Enrollment 


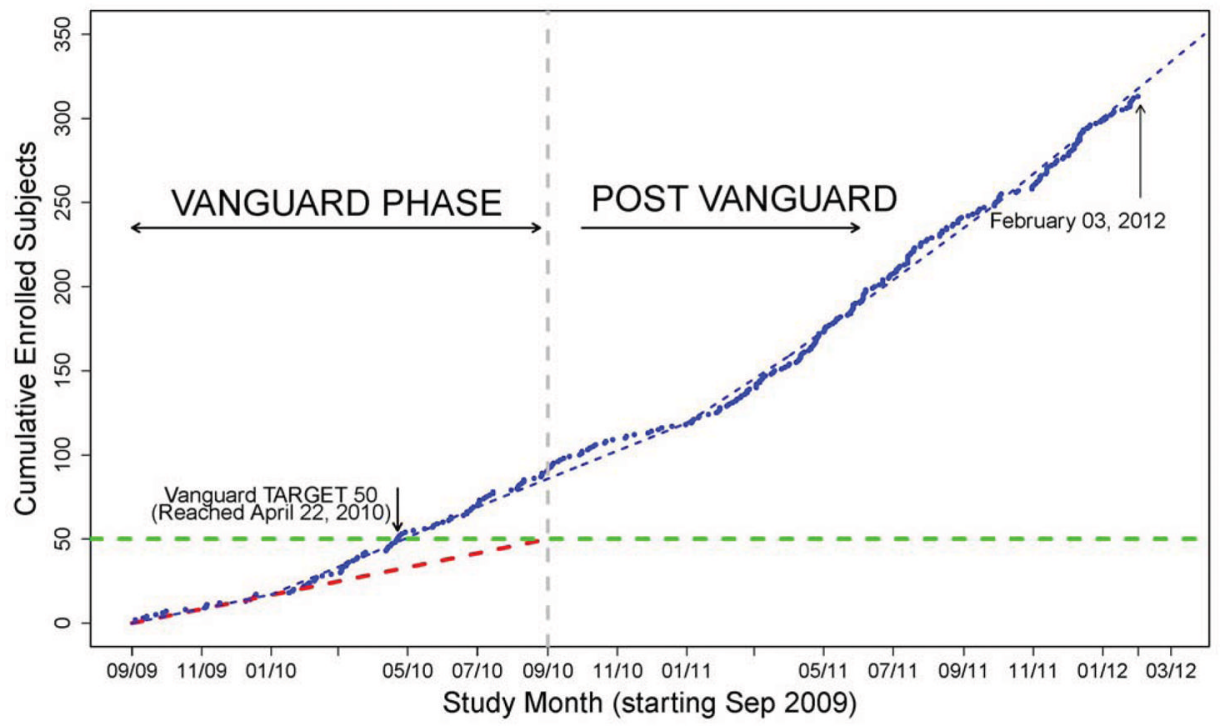

Figure 3.

THAPCA Current Enrollment (changes in the slope of the dashed line represent an increase in the target enrollment as recruitment ramped up and additional sites were added) 


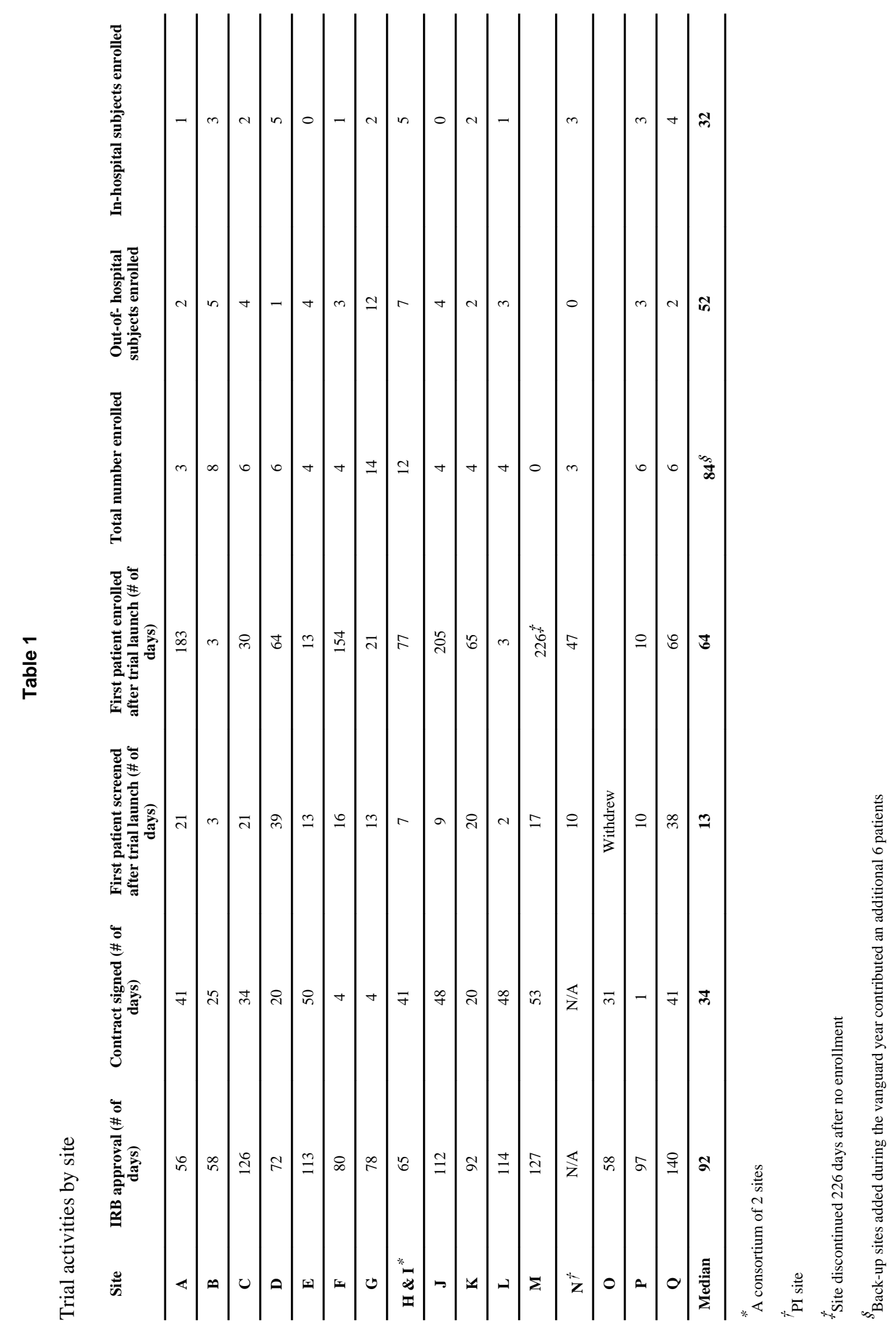

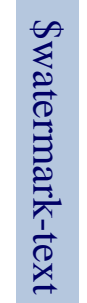


Table 2

Protocol changes during the vanguard phase

\begin{tabular}{|l|l|l|}
\hline Date & Protocol Amendment & Days to IRB approval \\
\hline & & Median (range) \\
\hline $6 / 29 / 2009$ & Simplification of protocol to decrease data collection and clarify temperature monitoring & $36(10-127)$ \\
\hline $10 / 22 / 2009$ & $\begin{array}{l}\text { Exclusion criterion changed to allow THAPCA- eligible subjects to be co-enrolled in other select } \\
\text { interventional studies }\end{array}$ & $34(7-104)$ \\
\hline $12 / 28 / 2009$ & $\begin{array}{l}\text { Exclusion criterion changed to allow children with previous cardiac arrests to be enrolled into the } \\
\text { trial. }\end{array}$ & $29(11-77)$ \\
\hline $5 / 11 / 2010$ & Incorporation of a family brochure and short consent form. & $43(24-176)$ \\
\hline
\end{tabular}

